Skin

Appendage

Disorders
Skin Appendage Disord 2019;5:283-287

DOI: 10.1159/000497392
Received: December 26, 2018 Accepted: January 30, 2019

Published online: March 22, 2019

\title{
Are Preferred Scalp Locations for Alopecia Areata Patches a Clue to Neuronal Etiology?
}

\author{
Margit Juhasz Natasha Atanaskova Mesinkovska \\ Department of Dermatology, University of California, Irvine, Irvine, CA, USA
}

\section{Keywords}

Alopecia · Hair loss · Alopecia areata · Scalp · Location ·

Nerve supply · Vascular supply

\section{Abstract}

Background: Alopecia areata (AA) is an autoimmune disease causing hair loss in $2 \%$ of the population. Anecdotally, hair specialists report that patches localize to the scalp periphery. Changes in sensory innervation and/or scalp vasculature may play a role in the development and localization of alopecic patches. Objective: To evaluate the most common locations of initial alopecic scalp patches. Materials and Methods: A retrospective chart review, with comprehensive evaluation of clinical photographs, was conducted from July 2016 to June 2018 to include AA patients $(n=112)$. Clinical data was collected on gender, age, race, time until presentation at the clinic, and areas of hair loss on initial presentation. Results: The most common areas of initial AA patches in both females and males were the occiput (49 vs. $48.5 \%$ ), parietal (46.9 vs. $21.2 \%)$, vertex ( 26.5 vs. $18.2 \%$ ), and frontal (24.5 vs. $18.2 \%$ ) regions; $26.8 \%$ of patients present with either alopecia totalis or universalis. Limitations: This is a single-center study with underrepresentation of minority races.
Conclusion: AA patches most commonly present on the occiput of the scalp in both female and male patients. Cervical spine nerves $C 3$ and $C 2$ supply sensory innervation and the occipital artery supplies blood to this area.

(c) 2019 S. Karger AG, Basel

\section{Introduction}

Alopecia areata (AA) is an autoimmune, inflammatory disease that selectively attacks the hair follicle, causing reversible hair loss. Disease severity ranges from affecting specific areas of the scalp (AA), to the entire scalp (alopecia totalis $[\mathrm{AT}]$ ) and even the body (alopecia universalis [AU]). Although this autoimmune disease affects approximately $2 \%$ of the population, large knowledge gaps still remain regarding its pathogenesis. Whilst anecdotal reports describe patients presenting with initial patches that favor the periphery of the scalp, there has been no peer-reviewed evidence supporting these claims.

Since the 1880s, animal models have suggested that AA may be associated with nervous system dysfunction or neurogenic inflammation. In the 1970s, research groups in Europe hypothesized a correlation between

\section{KARGER}

(c) 2019 S. Karger AG, Basel

E-Mail karger@karger.com

www.karger.com/sad
Margit Juhasz

Department of Dermatology, University of California, Irvine

843 Health Sciences Road

Irvine, CA 92697 (USA)

E-Mail mjuhasz@uci.edu 
sensory innervation and AA foci; however, no further research was completed to strengthen this association $[1,2]$. Multiple studies have since demonstrated differences in AA scalp sensory perception, as well as changes in levels of neuropeptides, neurotrophins, and neuropeptide-degrading enzymes [3-8].

Just as alterations in specific sensory nerve fibers may affect AA foci localization, another hypothesis may be that changes in the microvasculature of the scalp may influence disease location. Starting in the 1960s, researchers in Japan demonstrated structural changes in AA capillary beds, with electron microscopy images showing decreased capillary lumens in AA-affected scalp $[9,10]$. Other possible changes in AA microvasculature anatomy include small vessel vasospasm and dermal lymphatic dilation, as demonstrated in a recent $\mathrm{C} 3 \mathrm{H} / \mathrm{HeJ}$ graft-donor mouse model of AA [11, 12].

In this study, the authors revisit the concept of whether alopecic patches have a tendency to localize to specific areas of the scalp on initial presentation. Using a retrospective chart review, including clinical photographic assessment, from a single academic center, we demonstrate that patients with AA most commonly present with patches of the occipital and/or parietal scalp.

\section{Materials and Methods}

Chart review and evaluation of clinical photographs from the records of one faculty dermatologist specializing in hair disorders was conducted from July 2016 to July 2018. Demographic information collected included age, gender, and race; clinic notes were reviewed to discern information regarding alopecia disease including diagnosis (AA, AT, AU), time elapsed from initial spot to clinical presentation, and location of scalp alopecic patches on presentation. Data was collected and analyzed in Excel (Microsoft Office, Seattle, WA, USA).

\section{Results}

In the period from July 2016 to July 2018, 112 patients were identified with a diagnosis of AA, AT, or AU; 82 patients (73.2\%) presented with a diagnosis of AA and 30 (26.8\%) with AT/AU. Sixty patients were female and 52 were male; the majority of patients racially identified as "White" $(n=69)$. The average age of patients was $38.3 \pm$ 17.0 years (age range: $5-80$ years). The average time until presentation at the clinic was $7.5 \pm 11.8$ years (range: 1 month to 46 years), but 10 patients had no record of length of time of disease prior to presentation (Table 1).
Table 1. Alopecia areata patients

\begin{tabular}{lc}
\hline Gender, $\%$ & \\
Female & 53.6 \\
Male & 46.4 \\
Mean age, years & $38.3 \pm 17.0$ \\
Female & $37.9 \pm 16.5$ \\
Male & $38.7 \pm 17.8$ \\
Race, \% & \\
White (including Middle-Eastern) & 62.5 \\
Asian & 16.1 \\
Other/mixed & 14.3 \\
Hispanic & 3.6 \\
Black & 2.7 \\
Diagnosis, \% & \\
AA & 73.2 \\
AT/AU & 26.8 \\
Disease duration at presentation, years & $7.5 \pm 11.8$ \\
Female & $8.8 \pm 13.3$ \\
Male & $6.2 \pm 9.9$
\end{tabular}

AA, alopecia areata; AT, alopecia totalis; AU, alopecia universalis.

Most commonly, AA patients initially presented with alopecic patches on the occiput of the scalp (48.8\%), followed by patches in the parietal regions (42.7\%), the vertex/crown $(23.2 \%$,), the frontal scalp (22.0\%), the temporal region (11.0\%), and diffuse pattern (9.8\%). Other locations and patterns of initial hair loss included classic ophiasis pattern with hair loss encompassing the entire periphery of the scalp (2.4\%), as well as eyelash/eyebrow (6.1\%) and/or beard (1.2\%) only (Fig. 1). AU/AT patients were not included in this analysis as we were unable to confirm by history or photographic evidence the exact locations in which patches initially occurred before progression to hair loss of the entire scalp.

Subgroup analysis by gender revealed that males were more likely to present with AT/AU (36.6\%) compared to females (18.3\%). In female and male patients with AA, patches were most likely to occur in the occiput (49.0 vs. $48.5 \%)$, parietal ( 46.9 vs. $21.2 \%)$, vertex ( 26.5 vs. $18.2 \%$ ), and frontal (24.5 vs. $18.2 \%)$ regions of the scalp (Fig. 2).

\section{Discussion}

This retrospective chart review demonstrates that AA patches in the scalp are most often initially located in the occipital and parietal regions. However, it is still not clear as to why those areas of the scalp are most commonly af- 


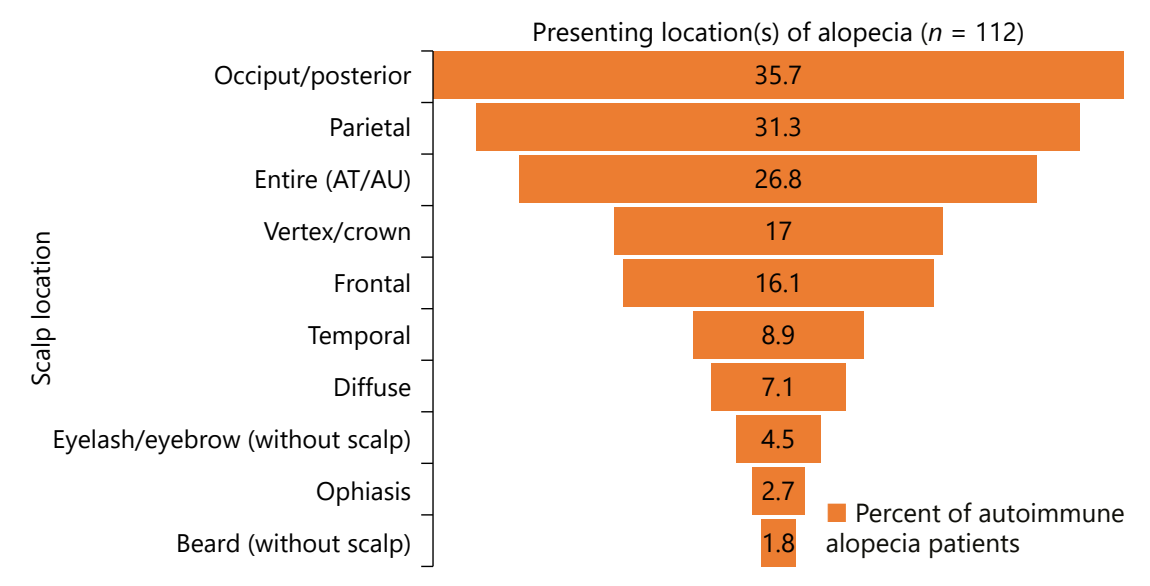

Fig. 1. Location of initial presentation of alopecia in patients.

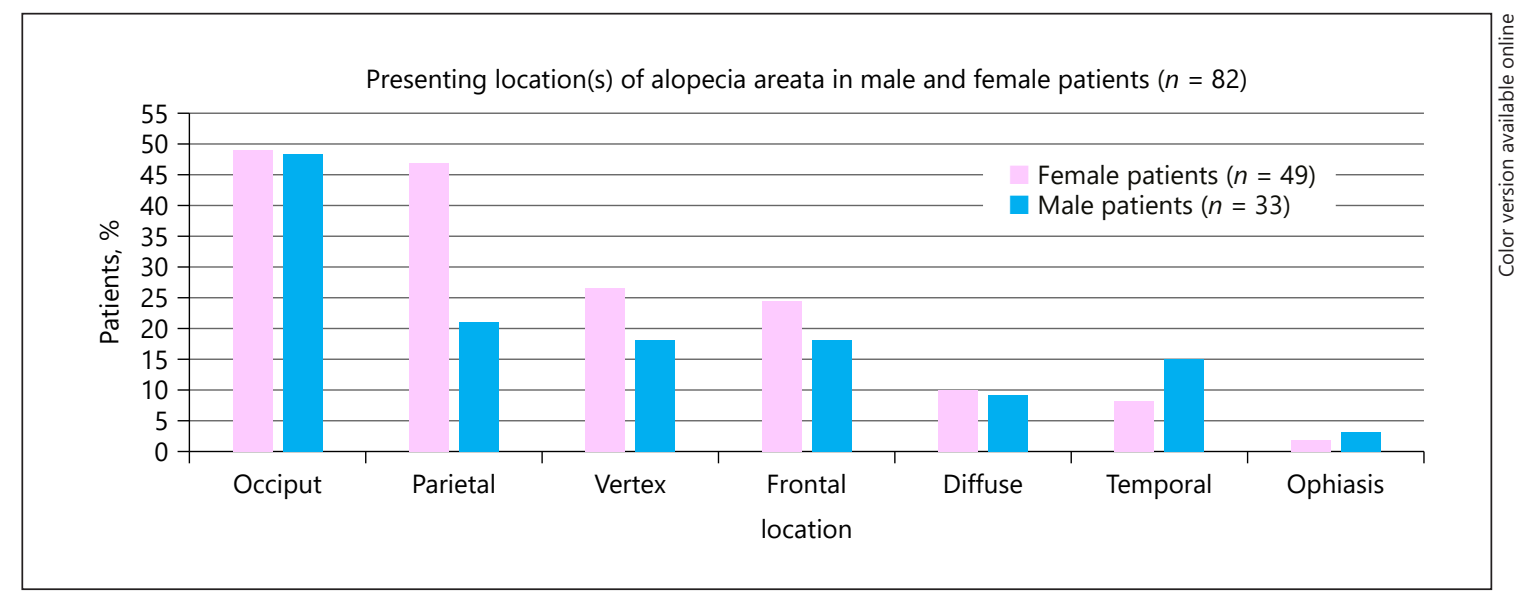

Fig. 2. Subgroup analysis of the presenting location of alopecia areata in female and male patients.

fected. It is possible that dysfunction of sensory innervation may play a role in disease development, especially considering many patients describe tingling or burning of their scalp prior to hair loss $[4,13]$. An early animal study in cats from 1886 suggested a neurogenic pathogenesis for AA; when posterior roots of the cervical spinal nerves originating from $\mathrm{C} 2$ were severed, animals developed characteristic AA patches [14]. A study conducted in Germany in 1970 suggested that $93.9 \%$ of alopecic patches in humans are located in a region of peripheral sensory innervation, with $67 \%$ originating from the second cervical segment [2]. The sensory innervation of the occipital scalp derives from the greater occipital and lesser occipital nerves that are branches of cervical spinal nerves originating from $\mathrm{C} 3$ and $\mathrm{C} 2$, respectively. The parietal area is innervated by the auriculotemporal nerve originating from the third branch of the trigeminal nerve (fifth cranial nerve $[\mathrm{CN} \mathrm{V}]$ ), while the temporal and frontal scalp regions are innervated by the second and first branch of $\mathrm{CN} \mathrm{V}$, respectively. These early results reflect our findings that the occiput is the most commonly affected AA area with sensory innervation in part originating from $\mathrm{C} 2$.

A decrease in neuropeptide expression may affect cutaneous nerves surrounding blood vessels, causing chang- 


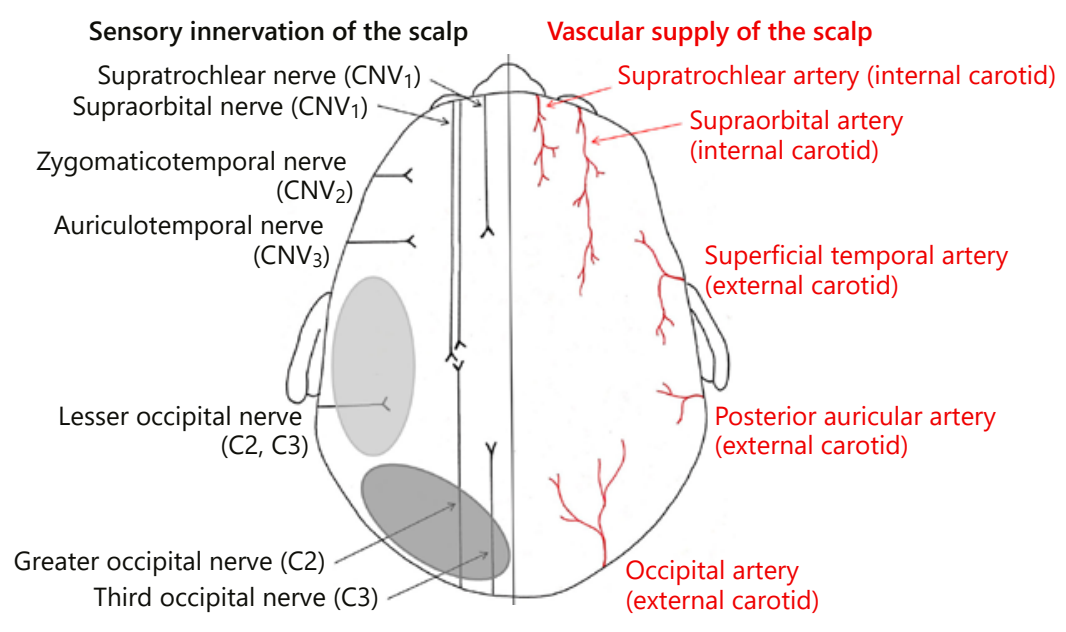

Fig. 3. Schematic depicting important neuronal and vasculature structures of the scalp [17]. Disturbed sensory innervation originating from cervical spinal nerves C2 and C3 may contribute to AA patches of the occipital (dark grey) and parietal (light grey) scalp.

es in microvasculature, such as decreased blood flow [5]. It is easy to hypothesize that lack of blood flow and decreased oxygen supply to the hair follicle may play a role in AA development. Further investigations into possible neurotrophic causes of AA have reported interesting results. Compared to healthy individuals, AA patients demonstrate decreased iontophoretic pilocarpine stimulation of eccrine glands [4], and require increased mean current intensity to evoke sensation from C-fibers [3].

In addition, dysregulation of neuropeptides, proteins important in homeostasis and tissue remodeling, may lead to AA development. Neuropeptides contribute to hair follicle growth and epidermal proliferation. These neurologic signal molecules may also lead to downstream effects on immune cells such as stimulating CD8+ T-cell apoptosis [15]. Mouse models demonstrate higher levels of substance P-immunoreactive nerve fibers in affected skin causing subsequent mast cell degranulation [6]. Although substance P-immunoreactive nerve fibers are increased, the actual concentration of substance $\mathrm{P}$, as well as calcitonin gene-related peptide, in scalp biopsies of AA patients is decreased due to increased expression of neuropeptide-degrading enzymes, such as neutral endopeptidase, in AA patients $[4,5,7,13]$.

Less research has been conducted to determine whether there are changes in vessels in AA-affected regions, however there are reports of luminal narrowing, vaso- spasm, and decreased overall blood flow $[9,10,12]$. Histologic analysis of AA scalp shows diminished capillary loops, branch-like spreading arterioles (candelabra arterioles), and a distorted hair follicle capillary network with loss of transverse anastomoses. Intralesional corticosteroid treatment of alopecic patches results in restoration of normal vasculature, as well as hair regrowth [16]. The vasculature of the occipital and parietal areas of the scalp arise from the external carotid artery with direct supply through the occipital artery and the posterior auricular artery, respectively. The temporal region is supplied by the zygomaticotemporal artery and the frontal scalp by the supratrochlear and supraorbital arteries, all branches of the internal carotid artery (Fig. 3) [17]. It is possible that changes in oxygen, nutrient, growth factor, and inflammatory marker delivery to the hair follicle may play a role in AA pathogenesis.

The findings presented in this paper raise new questions regarding AA and the potential role of nerve conduction studies, radiologic imaging of the neck for spinal nerve compression, or vascular studies using Doppler in early detection of disease. Characterization of nerve or vessel abnormalities on histology may provide further information on disease pathogenesis. This study is an attempt to establish a pattern and create a hypothesis. As it is a retrospective review of electronic medical records, data collection was limited by the accuracy and availabil- 
ity of patient charts. Patients with moderate to severe AA may be overrepresented in this population, as they are primarily evaluated by a dermatologist specializing in the diagnosis and treatment of hair diseases (N.A.M.). Furthermore, minority races such as Hispanic/Latino and Black Americans are underrepresented in our patient population when compared to national averages. Statistical analysis in this study was completed using only the data that was available at the time of chart review.

\section{Conclusions}

AA is an important cause of hair loss that clinicians to should be able to diagnose. This study demonstrates that AA patches of the scalp are most likely to occur in the occiput and parietal regions; subgroup analysis by gender reveals the same pattern. Whilst the pathogenesis of AA remains unclear, it is possible that either changes in sensory innervation or scalp vasculature may play a role in the localization of alopecic patches to these areas. Future directions should include a multicenter retrospective medical record review to determine whether these results are reproducible on a larger scale, with multiple patient populations, and further prospective cohort studies to determine whether we can predict AA disease course based on initial clinical presentation. We invite our colleagues to help us achieve these goals.

\section{Statement of Ethics}

This retrospective chart review was reviewed and approved by the Institutional Review Board of the University of California, Irvine.

\section{Disclosure Statement}

The authors have no conflict of interest to declare. This article has no funding source.

\section{References}

1 Gay Prieto J, González G, Alvarez Uría-Rico M. [Hair follicle's innervation in generalized alopecias]. Actas Dermosifiliogr. 1974 SepOct; 65(9-10):477-84.

2 Hönemann W, Höfer W. [Are there any connections between sensory innervation of the head and location of the alopecia areata foci?]. Dermatol Monatsschr. 1970 Jul;156(7):68390.

3 Farah RS, Farah RS, Junqueira Bertin AC, Guo H, Ericson ME, Hordinsky MK. Increased cutaneous stimulation is required for C-fiber sensory perception in alopecia areata: A double-blind study. J Am Acad Dermatol. 2016 Dec;75(6):1265-7.

4 Hordinsky MK, Kennedy W, WendelschaferCrabb G, Lewis S. Structure and function of cutaneous nerves in alopecia areata. J Invest Dermatol. 1995 May;104(5 Suppl):28S-9S.

5 Rossi R, Del Bianco E, Isolani D, Baccari MC, Cappugi P. Possible involvement of neuropeptidergic sensory nerves in alopecia areata. Neuroreport. 1997 Mar;8(5):1135-8.
6 Siebenhaar F, Sharov AA, Peters EM, Sharova TY, Syska W, Mardaryev AN, et al. Substance $\mathrm{P}$ as an immunomodulatory neuropeptide in a mouse model for autoimmune hair loss (alopecia areata). J Invest Dermatol. 2007 Jun; 127(6):1489-97.

7 Toyoda M, Makino T, Kagoura M, Morohashi M. Expression of neuropeptide-degrading enzymes in alopecia areata: an immunohistochemical study. Br J Dermatol. 2001 Jan; 144(1):46-54.

8 Winkelmann RK, Jaffe MO. Nerve network of the hair follicle in alopecia areata. Arch Dermatol. 1960 Nov;82(5):750-3.

9 Masumizu T. [Pathophysiological studies on alopecia areata. I. The so-called capillary picture in the foci of alopecia areata]. Nihon Hifuka Gakkai Zasshi. 1961 Apr;71:345-63. Japanese.

10 Mijailović B, Spasić P, Janković D, Karadaglić D, Stanković K, Bursać P. [Electron microscopy of blood vessels in alopecia areata]. Vojnosanit Pregl. 1989 Mar-Apr;46(2):105-7.
11 Sundberg JP, Pratt CH, Silva KA, Kennedy VE, Stearns TM, Sundberg BA, et al. Dermal lymphatic dilation in a mouse model of alopecia areata. Exp Mol Pathol. 2016 Apr;100(2):332-6.

12 Vamos L. [Data on vascular spasm in the pathogenesis of various cases of alopecia areata]. Borgyogy Venerol Sz. 1961 May;37:10610. Hungarian

13 Hordinsky MK. Overview of alopecia areata. J Investig Dermatol Symp Proc. 2013 Dec; 16(1):S13-5.

14 Joseph M. Experimentalle Untersuchungen uber die Aetiologie der alopecia areata. Derma. 1886;5:483. German.

15 Botchkarev VA. Neurotrophins and their role in pathogenesis of alopecia areata. J Investig Dermatol Symp Proc. 2003 Oct;8(2):195-8.

16 Popchristov P, Konstantinov A, Obreshkova E. The blood vessels of the scalp in patients with alopecia areata before and after corticosteroid therapy. Br J Dermatol. 1968 Nov;80(11):753-7.

17 Netter F. Atlas of human anatomy. 7th ed. Amsterdam: Elsevier; 2019. 